FACULTAD DE EDUCACIÓN

"PERFIL PROFESIONAL DEL INGENIERO INDUSTRIAL Y COMERCIAL EN UNA UNIVERSIDAD PRIVADA: PERCEPCIONES DE ACADÉMICOS Y EMPLEADORES"

Tesis para optar el grado académico de Doctor en Educación

ANTONIO TACCHINO DEL PINO

Lima - Perú

2016 
A mi madre que sabía que llegaría, A Giannina por su amor, siempre. 
Asesor:

Jorge Rodriguez Sosa 


\section{Índice de Contenido}

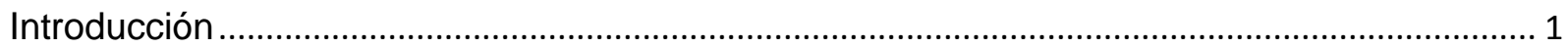

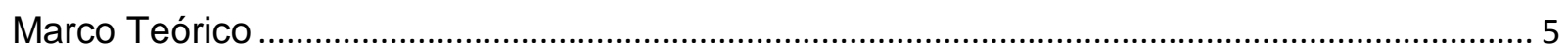

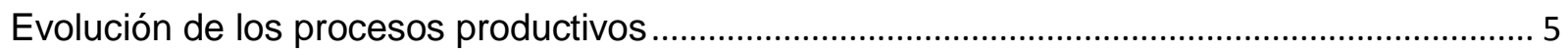

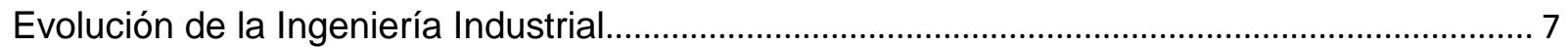

Las competencias profesionales en la enseñanza de la Ingeniería Industrial........................... 9

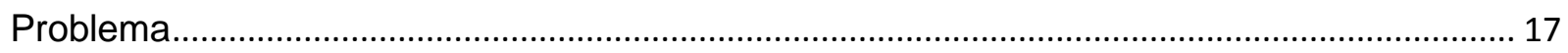

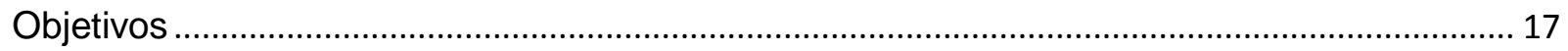

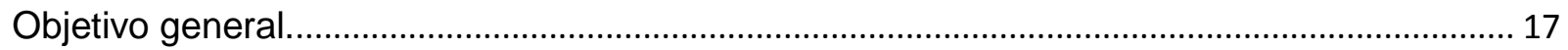

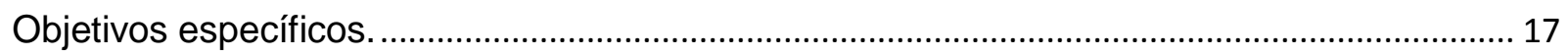

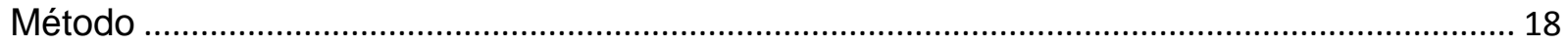

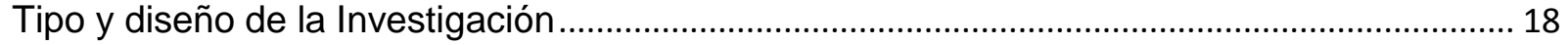

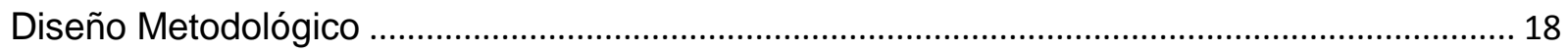

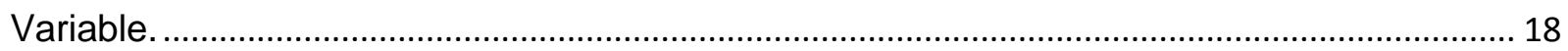

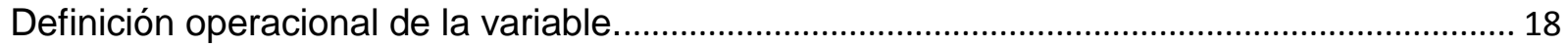

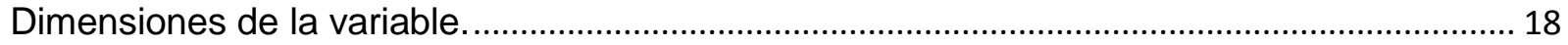

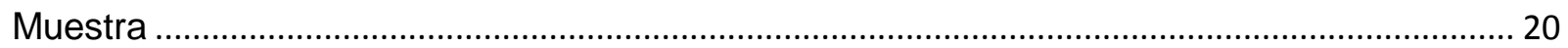

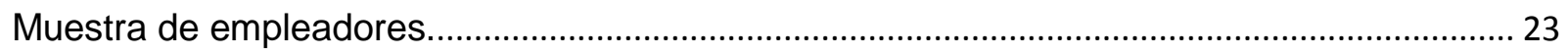

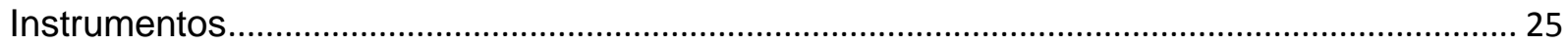

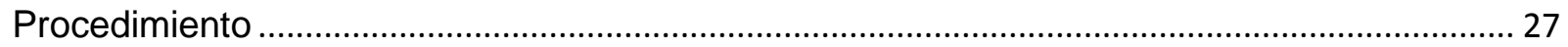

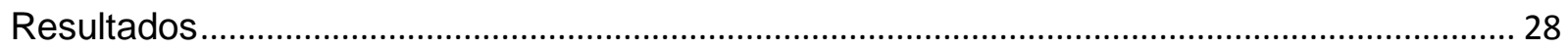

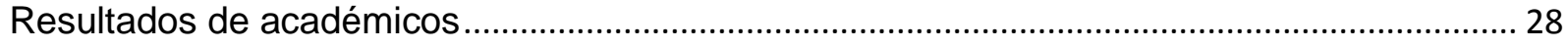

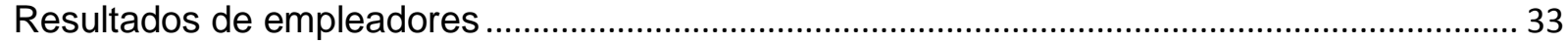

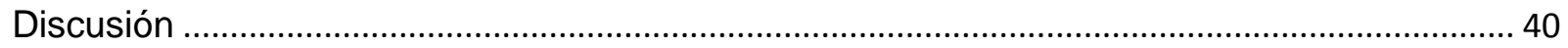

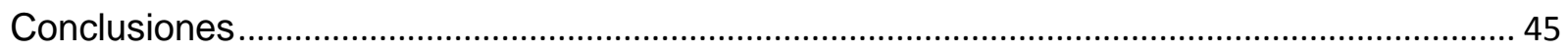

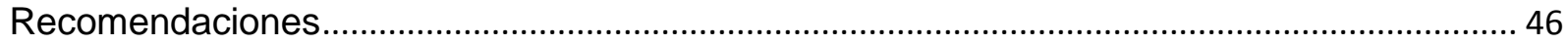

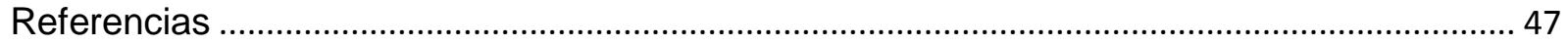

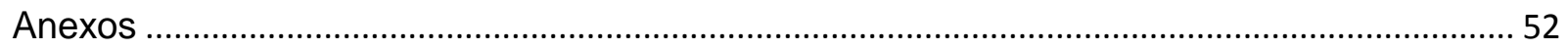

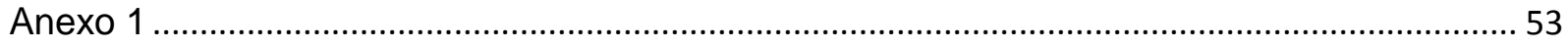

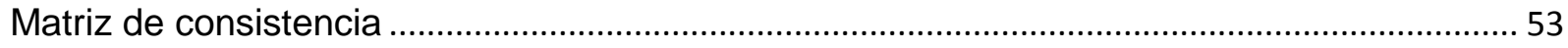




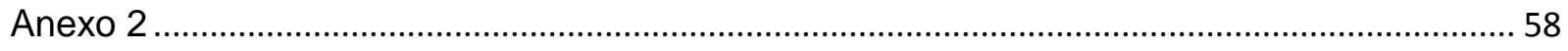

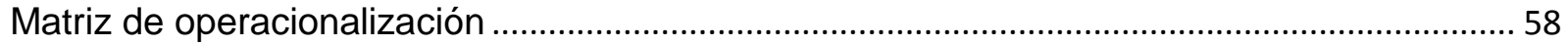

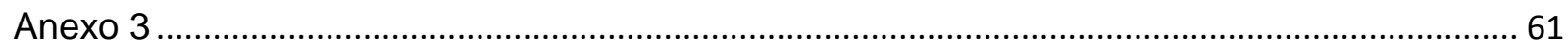

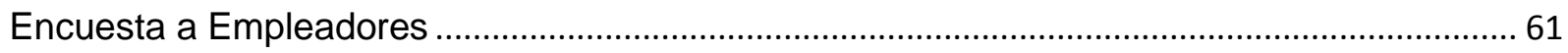

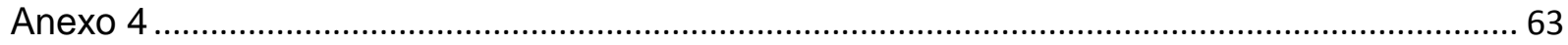

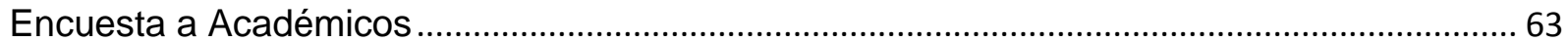




\section{Índice de Tablas}

Tabla 1. Objetivos de primer y segundo nivel de syllabus de CDIO ...................................... 12

Tabla 2. Elementos de competencia del IPMA ......................................................................... 13

Tabla 3. Tuning 2007 - Competencias genéricas acordadas para América Latina................. 14

Tabla 4. Dimensiones e indicadores de la variable. ................................................................. 19

Tabla 5. Cargas Factoriales del modelo tridimensional de competencias ............................... 26 


\section{Índice de Figuras}

Figura 1. Distribución de la muestra por profesiones ............................................................. 20

Figura 2. Distribución de profesiones según género ……………………………………….... 21

Figura 3. Distribución de profesiones según edad ...................................................................... 21

Figura 4. Años de experiencia en docencia universitaria repartida por sexo ............................ 22

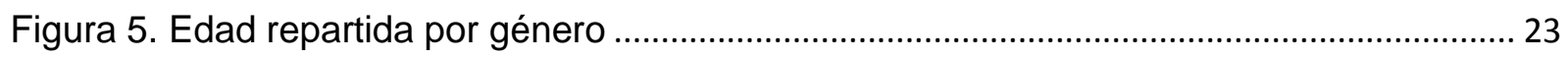

Figura 6. Distribución de la muestra por sector de empleadores ............................................... 24

Figura 7. Distribución de la muestra por cargo de empleadores ................................................. 24

Figura 8. Importancia de la matemática, física y química en el ámbito profesional ................. 28

Figura 9. Importancia del método y medición del trabajo .......................................................... 29

Figura 10. Género y percepción de competencias en la muestra de académicos .................... 30

Figura 11. Edad y percepción sobre competencias en la muestra de académicos................... 31

Figura 12. Experiencia docente y percepción sobre competencias en la muestra de académicos

Figura 13. Experiencia docente, edad y percepción de competencias en la muestra de académicos

Figura 14. Género y percepción de competencias en la muestra de empleadores

Figura 15. Sector donde se desempeñan empresarios y percepción de competencias en la muestra de empleadores.

Figura 16. Profesión del empresario, sector y percepción de competencias en la muestra de empleadores.

Figura 17. Profesión del empresario, sector, género y percepción de competencias en la muestra de empleadores. 


\section{Resumen}

El propósito del estudio fue describir las percepciones de académicos y empleadores sobre el perfil profesional del Ingeniero Industrial y Comercial de una universidad privada de Lima, así como establecer consistencias y diferencias en las percepciones de ambos tipos de informantes. Es un estudio descriptivo simple que trabajó con una muestra de 100 informantes, (50 académicos y 50 empleadores), a quienes se aplicó un cuestionario de 15 preguntas. Los resultados nos dicen que, en el marco de una percepción favorable tanto de académicos como de empleadores sobre las competencias que componen el Plan de Estudios observado, la opinión mayoritaria da prioridad a las llamadas competencias generales como las de comunicación integral (que incluye el uso de un segundo idioma), gestión adecuada de recursos y pensamiento crítico, como competencias de base para asegurar procesos adecuados de toma de decisiones. Las competencias técnicas, propias de la carrera, también son percibidas como importantes, pero en menor medida que las anteriores. Así, es necesario poner mucho énfasis en desarrollar de habilidades blandas (soft skills) como trabajar en equipo, trabajar con equipos multidisciplinarios, adaptarse a los cambios y reconocer la necesidad del aprendizaje permanente.

Palabras clave: competencias, perfil profesional, Ingeniería Industrial. 


\begin{abstract}
The purpose of the research was to describe the perceptions of members of academia, on the one hand, and employers, on the other, with regard to the professional profile of an Industrial and Commercial Engineer of a private university in Lima, as well as establishing consistencies and differences in the perceptions of both types of informants. It is simple descriptive research performed using a sample of 100 informants -50 members of academia and 50 employerswho were asked to respond to a questionnaire made up of 15 questions. The results show, firstly, that there was a favorable perception among both groups of informants regarding the skills that comprise the curriculum in question. Secondly, the majority of opinions give priority to general skills such as interpersonal communication (including the use of a second language), proper resource management and critical thinking, as basic to ensuring optimum decisionmaking processes. The technical skills within the major are also perceived as important, but to a lesser degree than the general skills. Hence, it is necessary to put major emphasis on the development of soft skills such as team work, ability to work with multidisciplinary teams, adaptation to change and recognition of the need for lifelong learning.
\end{abstract}

Key words: skills, professional profile, Industrial Engineering. 
